
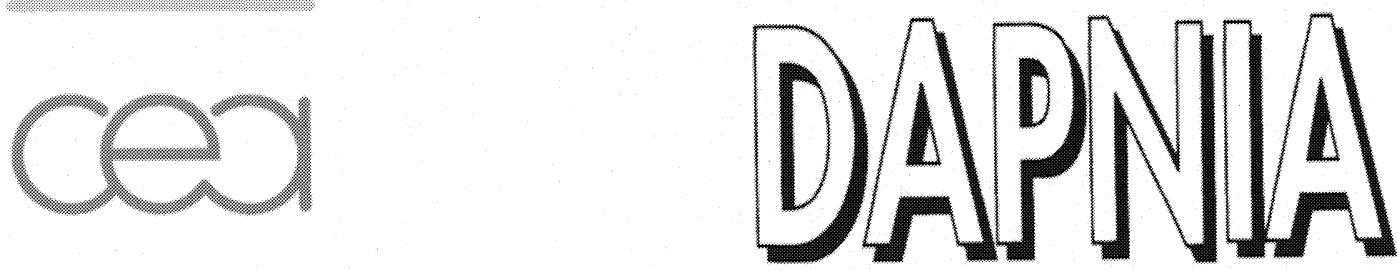

CERN LIBRARIES, GENEVA

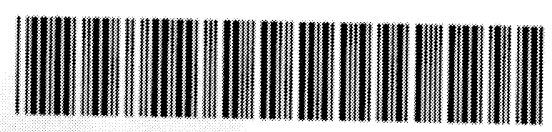

CM-P00058548
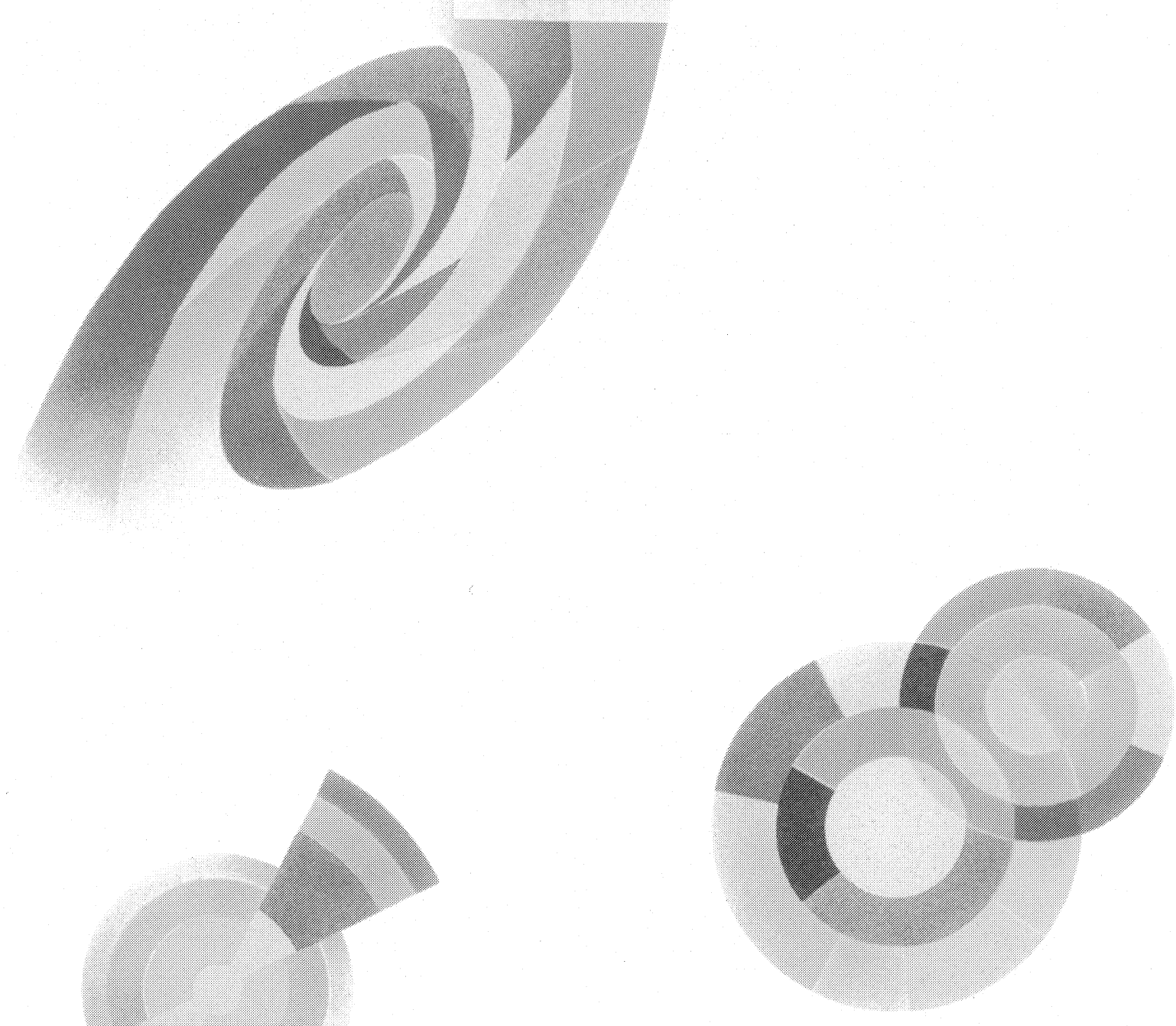

DAPNIA-04-508

$04 / 2005$

Calibrations of two alignment sensors used in the ATLAS barrel muon spectrometer.

J.C. Barriere, O. Cloue, B. Duboue, V. Gautard, C. Guyot, M. Fontaine, P. Perrin, P. Ponsot, Y. Reinert, J.P. Schuller, P. Schune

Talk given at the IEEE 2004 Nuclear Sctence Symposium and Medical Imaging Conference, Rome (taly), October 16-22, 2004

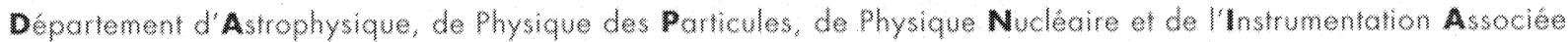




\title{
CALIBRATIONS OF TWO ALIGNMENT SENSORS USED IN THE ATLAS BARREL MUON SPECTROMETER
}

\author{
J.-Ch. Barrière, O. Cloué, B. Duboué, V. Gautard $\left({ }^{1}\right)$, C. Guyot, \\ M. Fontaine, P. Perrin, P. Ponsot, Y. Reinert, J.-P. Schuller, Ph. Schune \\ CEA-Saclay, DAPNIA \\ F-91191 Gif-sur-Yvette Cedex, France
}

\begin{abstract}
ATLAS is a particle detector which will be built at CERN (Geneva) at the Large Hadron Collider (LHC) accelerator. The ATLAS barrel muon spectrometer is made up by 600 chambers positioned in three layers embedded in a toroidal magnetic field. Thus each muon track is detected by three muon chambers within a projective tower performing a sagitta measurement. In order not to deteriorate the sagitta measurement, the muon chamber position must be known within a tower with a spatial resolution of $30 \mathrm{um}$. To fultil these requirements, different optical alignment systems have been built. In the ATLAS barrel spectrometer, six different alignment types participate to the global alignment. Among them the PRAXIAL and REFERENCE sensors have been developed at Saclay.

A short introduction on the alignment of the experiment is given in the first section, the second and third sections are devoted to the PRAXIAL system calibration. The fourth section describes the REFERENCE alignment systems. The last part is related to the user interface that manages all alignment calibration benches.
\end{abstract}

\section{THE ALIGNMENT OF THE ATLAS MUON SPCTROMETER}

\subsection{The Muon Spectrometer}

The ATLAS experiment (see Fig. 1), is a detector that will be buit on the LHC accelerator $[1,2]$ at CERN [3]. The LHC will provide proton-proton interactions with a centre of mass energy of $14.10^{12} \mathrm{eV}_{\times}$One of the physics goals is to detect the hypothetic Higgs boson. Despite the fact that its existence is crucial for the particle physics Standard Model, it has not yet been observed. The Higgs particle may decay through two $Z^{\prime}$ particles each decaying into two leptons: e.g. muons or electrons. Thus the ATLAS spectrometer is of particular importance.

The muon momentum measurement in the ATLAS muon spectrometer aims at an accuracy of the muon sagitta of the order of $10 \%$ for 1 TeV muon. At this energy the resolution is dominated by chamber sagita resolution which is about 40um. Thus the sagitta resolution coming from (mis)algnment should not exced 30um. To fulfil this prectston, several alignment sensors have been designed.

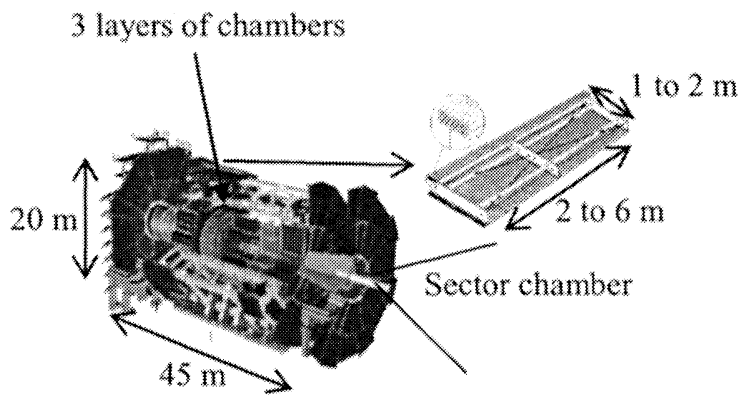

Figure 1: The ATLAS detector (right) and a muon chamber (left).

Finally the magnetic field is provided by eight individual coils (each $25 \mathrm{~m}$ by $5 \mathrm{~m}$ ) dividing the spectrometer in 16 sectors: 8 sectors made by so-called large muon chambers and 8 sectors made by so-called small chambers.

\subsection{The Barrel Alignment System}

The ATLAS barrel spectrometer alignment system is made of six different alignment types:

1. The IN PLANE alignment which measures chamber internal deformation.

2. The PROJECTIVE system which gives the chamber position within a tower.

3. The PRAXIAL system composed of wo parts:

3.1. The PROXIMITY part which gives the position of one chamber with respect to the neighbouring one.

3.2. The AXIAL part controls the saloon door effect of chambers relative position within a layer.

4. The REFERENCE system used to link a sector of chambers to the neighbouring sector and to the coll.

5. The CCC system which connects large chambers to small chambers since the lasts are not individually aligned with REFERENCE.

\footnotetext{
Corresponding author, emant: gautard(acea.fr, tel $+33(0) 169084596$.
} 
6. The BIR-BM system used on the feet region of the experiment due to special muon chambers layout.

All these alignment systems may be divided in two categories of individual optical sensor: the Rasnik system and the Sacam system which are described in next section.

The NNPLANE, PROJECTIVE, PRAXIAL and BIRBIM systems use the RASNIK optical sensors while the REFERENCE and CCC systems are based on Sacam optical sensors.

\subsection{The Rasnik Sensor}

The Rasnik sensor (Red Alignment System of NIKLIEF) has been developed by the NIKHEF institute in Amsterdam $[4,5]$. It measures the relative position between three elements: a modified chessboard pattern mask lightened by infrared LEDs, seen by a camera through a lens (see Fig. 2).

This optical system measures four parameters: the $2 \mathrm{D}$ transverse position with an accuracy of $\sim 2 \mu \mathrm{m}$; the optical magnification on the camera with an accuracy below $10^{-4}$ and finally the relative angle between the mask line and the camera pixels line with an accuracy of the order of sourad.

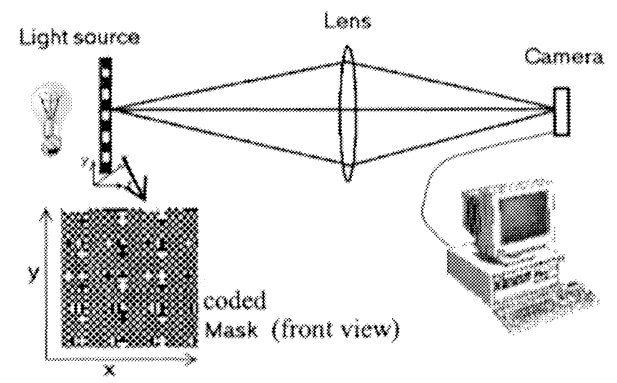

Figure 2: The RASNIK Sensor.

An online software called ICARAS arives the infrared LEDs and the camera, through a RS232 device. The image of the coded mask seen by the camera is then digitised through a frame-grabber card and the four coordinates of the system are computed.

\subsection{The Sacam Sensor}

The Sacam sensor consists of a camera embedded together with a lens in a mechanical box looking to a set of four illuminated holes. For some optical lines the illuminated holes can surround the lens of the embedded camera resulting to a situation where two cameras are facing to each other.

The measured quantities by the Sacam sensors are the two transverse angles with respect to the optical axis. Two other parameters are measured: the rotation angle of the illuminated holes with respect to the optical axis and the magnification of the reconstructed holes on the camera.
We developed an analysis module which determines these 4 parameters. In a first time, we calculate the centre of each spot by a barycentre method. To get the final accuracy a gaussian fit is performed and the spot centre is determined with an accuracy of -0.03 pixel. The relative angular resolution is about 5 urad for a range of about 18 mrad.

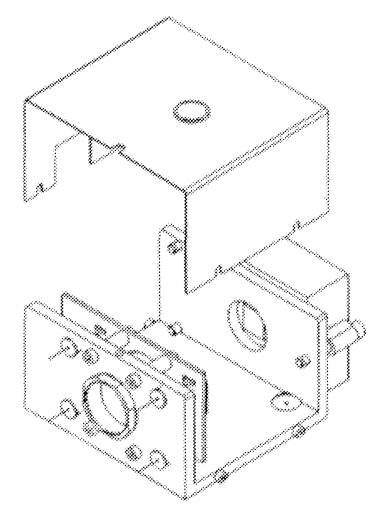

Figure 3: The Sacam sensor: a camera is embedded together with a lens in a mechanical box. Some sensors have also four illuminated holes surrounding the lens.

\section{PRAXIAL CALIBRATION BENCHES}

The PRAXIAL sensor is composed by two different sensors: the PROXIMITY and the AXIAL part (see section 1.2 and Fig.4). As we have to calibrate about 2000 PROXIMITY parts and 3000 AXIAL components we built two PC controlled calibration benches. They have been installed in an air ${ }^{\prime}$ conditioned room to avoid thermal variations during the calibration. In this section, we will concentrate on the PROXIMITY part: first present the PROXIMITY system, then its calbration, then the calibration bench (hardware and software) and lastly the results obtained up to now.

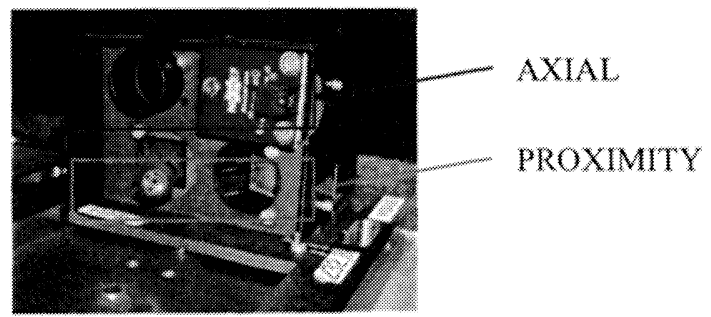

Figure 4: PRAXIAL sensor: PROXIMITY part (bottom) and the AXIAL part (top). For the PROXIMITY part the same detector is positioned on the neighbouring chamber. 


\subsection{The PROXIMITY system}

The PROXMMTY system, developed at Saclay 161, is composed of wo crossed RASNIK. The optical components are mounted on two mechanical supports each positioned on two neighbouring chambers (see Fig. 5).

As we have different distances between the chambers in the ATLAS experiment, we have sensors of different types. Indeed, for short distances $(-20 \mathrm{~mm}$ between neighbouning chambers) the two crossed RASNIK makes an angle of 25 degrees, while for large distances (up to $370 \mathrm{~mm}$ between neighbouring chambers) the opening angle is only 14 degrees. As the two crossed RASNIK are more and more parallel, the resolution on some translation is degraded (see section 2.4). Also the RASNIK magnification variation in the last configuration deteriorates the final sensor resolution.

Both RASNIK gives four measurements permitting to calculate the six degrees of freedom describing the relative position of one element with respect to the other one. The wanted resolution is $-10 \mathrm{~mm}$ for translations and -100 urad for rotations in a range of $\pm 5 \mathrm{~mm}$ and $\pm 5 \mathrm{mrad}$.

As it is impossible to mount the optical components with this accuracy, we have to perform a calibration of the PROXIMITY part.

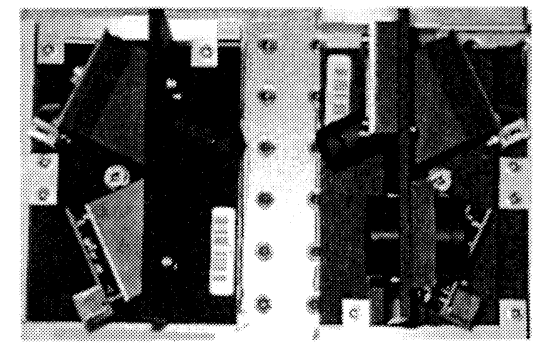

Figure 5: top view of the PROXIMITY part. The two crossed RASNIK component are clearly seen. In real life each mechanical support seats on two neighbouring chambers.

\subsection{The calibration principle}

The goal of the calibration is to determine a transfer matrix $\mathrm{P}$ used to compute the position between two neighbouring chambers. $P$ is a $6 \times 8$ matrix: 6 from the 6 degrees of freedom and 8 from the $2 \times 4$ RASNIK data.

In order to do that, one of the two sensor seats on a static plate while the other seats on a mobile plate. The mobile plate is then moved 64 times and at each position, precisely measured thanks to mechanical probes, the two RASNIK outputs are recorded. After this set of movements has been done, an analysis module fits the transfer matrix knowing the RASNIK measurements for each known position.

To check the transfer matrix calculation, another set of 55 random known movements is performed and a comparison of the transfer matrix calculation and the mechanical probes result is done. Thus, the final resolution of the sensor is estimated.

\subsection{The calibration bench}

First the hardware is described and in a second step the software is described [7].

\subsubsection{The hardware}

The complete calibration bench (see Fig 6 and 7 ) is composed of four main components.

First

1. A mechanical element, called ZEROPRAX, is used to define a common mechanical frame for the two PROXIMITY neighbouring sensors. This mechanical element is built with an accuracy of $5 \mu \mathrm{m}$ in order to have an absolute calibration.

Now for the calibration itself:

2. A set of 6 stages ( 3 for translations and 3 for rotations) are assembled to move the mobile sensor during the calibration.

3. Due to parasitic movements of the stages (yaw, titt) a set of 6 probes are used to measure the real displacement with an accuracy of $1 \mu \mathrm{m}$.

4. A set of temperature probes is installed on and around the bench. We record the temperature with the required accuracy $(0.5$ degree $)$.

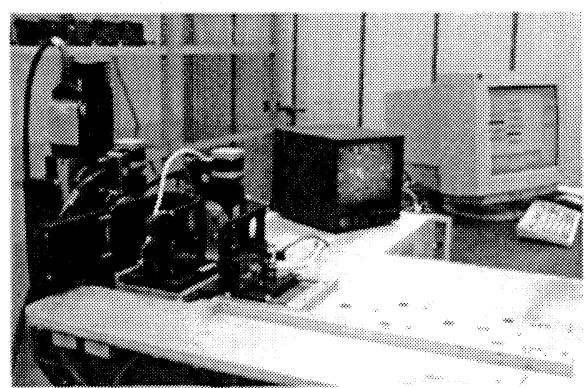

Figure 6: Overview of the calibration bench. One of the sensor seats on a mobile platform (left element) and the other one is fixed (right element).

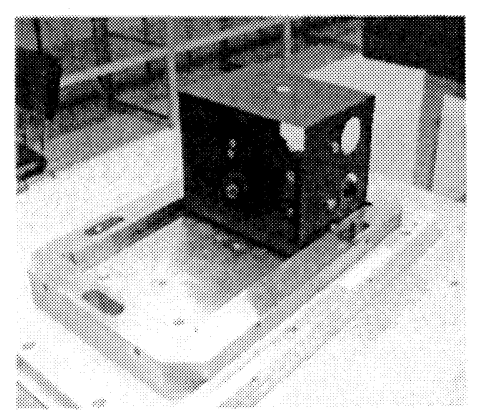

Figure 7: One of the two PROXIMITY sensor seats on perfect $(<5 \mu \mathrm{m})$ mechanical support (called

ZEROPRAX) in order to have an absolute calibration. 


\subsubsection{The software}

The software could be described in six steps:

1. A user friendly interface; one just click on the calbration button and all is automatically done.

2. The whole calibration hardware (probes, stages, sensors, etc.) is software controlled. We use RS232 and RS485 devices coupled with data acquisition cards. The thread computation has two advantages: i) we do not spend any CPU resources; ii) the signal is read instantaneously (e.g. bar code reading).

3. The different tasks must be ordered for example, we can't record the value of the position probe as long as the motor displacements are not ended.

4. Some software work together: e.g. the ICARAS software is triggered by the CALIPRAX software.

5. Some analysis modules are critical. The most important determines the transfer matrix coefficients. For that purpose, two methods have been compared: a linear fit and a chi2 minimisation. The linear fit has been chosen for its robustness even if the system is not linear. Another analysis module calculates the real movement according to the mechanical probes measurement.

6. Finally all data are stored both in text fles and in the ATLAS data base. The link to the database is an ODBC link.

This bench is used daily with no particular problem.

\subsection{The results}

Up to now, we have calibrated 700 PROXIMITY pairs. As we have different distances between ATLAS muon chambers, the PROXIMITY sensor resolution varies with this parameter (see section 2.1 ). We have a resolution better than 10 um in translations (see Fig 8 ) and 100 urad for rotations for sensors on chambers close to each other. For sensors on chambers with large distance in-between (up to $370 \mathrm{~mm}$ ) the resolution along this axis is deteriorated and is only $\sim 60 \mathrm{um}$.

We study the stability by calibrating successively the same PRAXIAL sensor. We found that the stability is below $3 \%$. We also test the reproducibility by calibrating the same PRAXIAL sensor at different times. We found that the reproducibility is below $6 \%$.

We conclude that these calibrations fulfil ATLAS requirements.
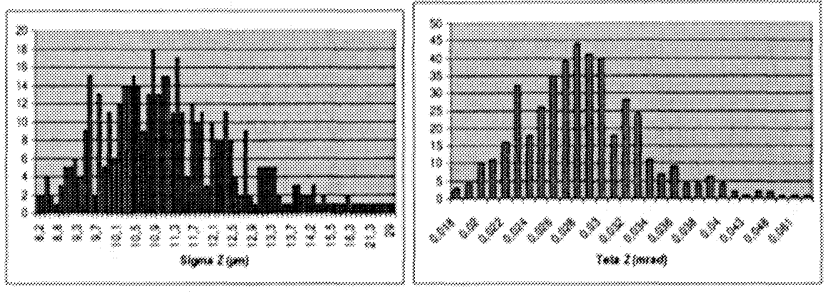

Figure 8: Resolution of 500 PROXIMITY sensors along $Z$ axis (left, in $\mu \mathrm{m}$ ) and on theta $Z$ (right, in milli-radian). In

ATLAS, $Z$ is the distance between two neighbouring chambers.

\section{OTHERS ALIGNMENT SYSTEMS}

In this chapter, we will tackle in a first part to the AXIAL alignment system and in a second part to the REFERENCE system.

\subsection{Axial system}

The AXIAL system is mandatory to control saloon door effect between chambers on the same layer. This uncertainty remains when using only PROXIMITY sensors which measure only locally relative chambers position.

This system has to measure only transverse position with a resolution of $10 \mu \mathrm{m}$ and rotation along its optical axis (see below) with a resolution of 200 urad.

The AXIAL is also based on a RASNIK sensor. Each component (i.e. mask, lens and camera) seats alone on top part of a PRAXIAL sensor (see Fig 4). Thus an AXIAL. system used three individual PRAXIAL support.

Like for the PROXIMITY system, it is impossible to mount the optical components with the required accuracy. So we have to perform a calibration independently of the calibration of the PROXMMTY elements: each AXIAL. optical component is calibrated individually with respect to two known blocks (see Fig. 9 for an example of a known mask block). These known blocks have been built using precise mechanical elements $(<5 \mathrm{um})$ and with an optical element at a known position ( $<54 \mathrm{~m}$, resulting from a specific calibration process). We have three known blocks: a mask, a lens and a camera.

In order to calbrate an AXIAL mask element we use two known blocks (lens and camera), positioned the three elements on a granite table. Thus with only one measurement the mask component is calibrated.

As for the previous calibration bench, this bench is software controlled and its output are stored in a data base. 


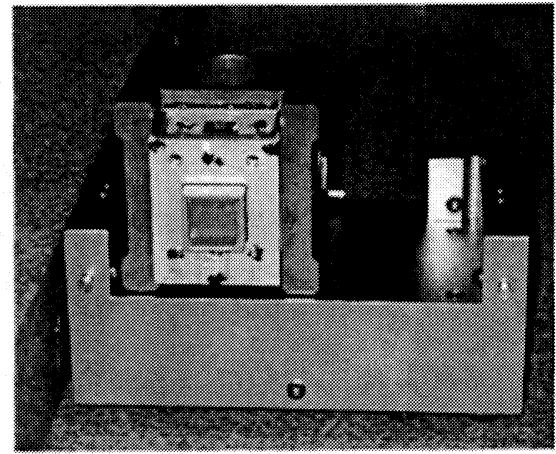

Figure 9: a known mask block used for the AXIAL calibration bench.

\subsection{The Reference system}

The ATLAS REFERENCE alignment system is required in order to correct for ambiguties coming from other alignment systems: mainly because RASNIK sensor magnification is a ratio of two distances and not an absolute distance measurement.

In ATLAS, the REFERENCE system measures the relative position of several chambers in different layers with respect to a common element: the reference plates glued on the toroidal magnet.

This system is based on the Sacam sensor (see section 1.4).

\section{GLOBAL SUPERVISOR}

The global supervisor controls all the steps: from sensor mechanical construction to calibration (see Fig 10) as control command, data base, etc.:

1. It reads bar code through a RS232 device. Then the sensor status is shown: built, checked, calibrated or other..

2. It is linked with the data base: e,g. when a user wants to build a PRAXIAL sensor, he begins by clicking on the supply button. The list of all goods appears and is automatically subtracted from the storage database.

3. When user clicks on the calibration button the CALIPRAX software is latnched.

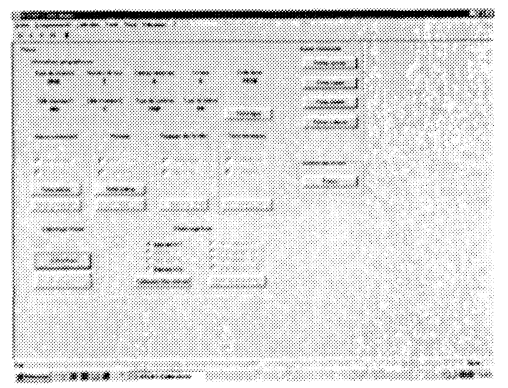

Figure 10: a view of the global supervisor
The global supervisor can be considered as a guide for the users and as a QAQC interface.

\section{CONCLUSION}

In this paper, we have presented the status of some alignment systems used in the ATLAS experiment.

In particular the following steps of the PROXIMITY system have been described: construction, calibration, QAQC procedure. The resolution of our sensors fulfils ATLAS requirements.

The AXIAL calibrations have now begun.

In the CERN 18 [8] test beam, 40 of our sensors are used like the REFERENCE software analysis package.

In parallel the final integration of ATLAS muon chambers has now begun at CERN.

\section{REFERENCES}

[1] http:/he, web.cern.ch/the

2] "ATLAS Technical Proposal", CERN/LHCC 9443 , 15 December 1994

"ATLAS Muon Spectrometer Technical Design Report", CERN/LHCC197-22, 31 May 1997 .

http//atlasinfo,cern.ch:80/Atlas/Welcome.html

[3] http//public.web.cern.ch/Public/Welcome html

[4] http://www.nikhef.nl

[5] H. van der Graaf et $a$ l. "Rasnik, an alignment system for the ATLAS MDT barrel muon chambers". Technical system description, ET38110, April 2000. See also: http://www.nikhef.nl

[6] J* Ch. Barrière, O. Cloué, B. Duboué, $V$. Gautard, P. Graffin,$C_{8}$ Guyot, P. Perrin, P. Ponsot, $Y$, Reinert, J. $P$. Schuller, Ph. Schune. "An optical sensor for the alignment of the ATLAS muon spectrometer", presented at the 3rd Beame Conference on New Developments in Photodetection, Beaune (France), June 2002.

[7] V. Gautard, O. Cloue: "PC based Control system of a calibration bench for the ATLAS experiment", presented at the 4th Int. Workshop on PCaPAC 2002, Frascati (talie), October 2002

81 "Optical alignment system for the ATLAS muon spectrometer", presented by F. Bauer at this conference. 